\section{MS4-P10 Mask-based approach to phasing single-particle diffraction data}

Alexandre Urzhumtsev ${ }^{1,2}$, Natalia L. Lunina ${ }^{3}$, Taniana E. Petrova $^{3}$, Manfred W. Baumstark ${ }^{4}$, Vladimir Y. Lunin ${ }^{3}$

\section{IGBMC, Illkirch, France}

2. Université de Lorraine, Nancy, France

3. IMPB RAS - Branch of KIAM RAS, Pushchino, Russia

4. University of Freiburg, Germany

email: sacha@igbmc.fr

We suggest a new ab initio phasing method [1] which is particularly appropriate for diffraction data from a single particle. A test with a known structure resulted in phases that have $97 \%$ correlation with the exact ones in the full $25 \AA$ resolution shell. Currently such data are phased mostly by 'solvent-flattening type' iterative procedures [2] based on the object's property to have a finite support. In our method, in addition to this feature, we use the connectivity of biological macromolecules and prior information in the form of probability distributions. The random search procedures allow a wide exploration of configurational space of possible phase solutions.

Instead of the search for the phase sets giving connected images as used previously [3], we start from a random generation of connected binary envelopes of a given volume. For each envelope, we calculate its structure factors and accept the corresponding phase set only if the amplitudes are very similar to the experimental values. Typically, from near a million of envelopes generated a hundred phase sets are selected, their values are aligned to their common origin and averaged.

Recent advances in the X-ray diffraction experiments lead to a possibility to register X-rays scattered by large isolated biological particles in all directions. In practice, the original continuous diffraction pattern is sampled at grid points in reciprocal space. This make the phase problem equivalent to one for an imaginary crystal with the large unit cell containing a particle and a large volume of empty space ("solvent"). The smaller the sampling step, the larger the unit cell and the "solvent fraction". Using a known structure, we investigated the effect of the sampling step on the accuracy of the phase problem solution. Reducing the sampling step, it is shown that an expected improvement of the accuracy of the solution continues even beyond the Nyquist limit. On the other hand, the method is also efficient when the sampling corresponds to a unit cell which size is close to that occurring in crystallographic studies.

1. Lunin VY et al. (2016) Acta Cryst., D72, 147

2. Fienup J. (1982). Appl.Optics, 21, 2758

3. Lunin VY, Lunina NL, Urzhumtsev A. (2000) Acta Cryst., A56, 375

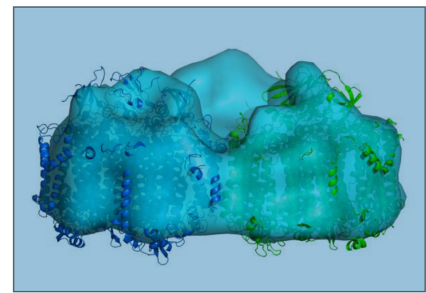

Figure 1. Image of trimer PS-I (Jordan et al. (2001) Nature, 411, 909) shown by $25 \AA$ resolution $a b$ initio phased Fourier synthesis calculated with the simulated magnitudes. Two of three monomers in the atomic model are shown (the $3^{\text {rd }}$ is removed for clarity).

Keywords: ab initio phasing, single particle, connected masks, pattern sampling 